\title{
Associations Between Homeostasis Model Assessment (HOMA) and Routinely Examined Parameters in Individuals With Metabolic Syndrome
}

\section{L. ŠTĚPÁNEK ${ }^{1}$, D. HORÁKOVÁ ${ }^{1}$, L. ŠTĚPÁNEK ${ }^{2}$, L. CIBIČKOVÁ ${ }^{3}$, D. KARÁSEK $^{3}$, H. VAVERKOVÁ ${ }^{3}$, M. NAKLÁDALOVÁ ${ }^{4}$, L'. JURÍČKOVÁ ${ }^{1}$, H. KOLLÁROVÁ ${ }^{1}$}

${ }^{1}$ Department of Public Health, Faculty of Medicine and Dentistry, Palacký University Olomouc, Czech Republic, ${ }^{2}$ Institute of Biophysics and Informatics, First Faculty of Medicine, Charles University, Czech Republic, ${ }^{3}$ Third Department of Internal Medicine - Nephrology, Rheumatology and Endocrinology, University Hospital Olomouc and Faculty of Medicine and Dentistry, Palacký University Olomouc, Czech Republic, ${ }^{4}$ Department of Occupational Medicine, University Hospital Olomouc and Faculty of Medicine and Dentistry, Palacký University Olomouc, Czech Republic

Received February 14, 2019

Accepted July 26, 2019

Epub Ahead of Print October 25, 2019

\section{Summary}

The aim of the study was to investigate whether routine clinical parameters, including visceral adiposity index (VAI) and atherogenic index of plasma (AIP), could become widely applicable predictors of insulin resistance (IR), evaluated using homeostasis model assessment (HOMA-IR, HOMA- $\beta$ ), with regard to presence of metabolic syndrome (MS). The study comprised 188 individuals identified to meet the MS criteria during regular health examinations and an equal number of age, sex-matched controls without MS. The strongest correlations were noted between HOMA-IR and waist circumference (WC) in the MS group ( $r=0.57$ ) as well as between HOMA-IR and alanine aminotransferase (ALT, $r=0.57$ ) or aspartate aminotransferase $(r=0.56)$ in the controls, with a statistical significance of $p<0.001$. In a multivariate linear regression model, the predictors of HOMA-IR were WC (linear coefficient $\beta=0.1, p<0.001$ ), ALT $(\beta=2.28, \quad p<0.001)$ and systolic blood pressure $(\beta=0.04$, $p<0.001)$. HOMA- $\beta$ was determined by WC $(\beta=1.97, p=0.032)$ and ALT $(\beta=99.49, p=0.004)$ and inversely associated with age $(\beta=-1.31, p=0.004)$. Neither VAI nor AIP were significant predictors. The presence of MS was significantly associated with both HOMA-IR and HOMA- $\beta$. These results indicate that WC and ALT appear to be reliable predictors of IR. Comprehensive assessment of these parameters may serve for estimating the level of IR.

\section{Key words}

Insulin resistance - Homeostasis model assessment - Visceral adiposity index $\bullet$ Atherogenic index of plasma $\bullet$ Waist circumference

\section{Corresponding author}

L. Štěpánek, Department of Public Health, Faculty of Medicine and Dentistry, Palacký University Olomouc, Hněvotínská 3, 77515 Olomouc, Czech Republic. E-mail: stepanek.ladislav@gmail.com

\section{Introduction}

Globally, chronic noninfectious diseases play an important role and increasingly contribute to overall mortality. There is therefore a growing need to search for and use new preventive methods. Metabolic syndrome (MS), grouping factors crucial for the development of diseases of affluence, has been defined as a single nosological entity, among others, to clearly identify individuals with high cardiovascular risk eligible for targeted preventive interventions (Paniagua 2016).

Obesity is a major clinical problem in developed societies. It is well known that obesity and MS are associated with increased morbidity and mortality (Matloch et al. 2016). According to some definitions, visceral obesity, as measured by waist circumference (WC), is stressed over other components of MS (Kassi et al. 2011). In the state of obesity, subjects already present insulin resistance (IR) and hyperinsulinemia, probably the first step of a dysfunctional metabolic 
system. Subsequently, other disorders develop gradually, in particular hyperglycemia, dyslipidemia and hypertension, that is, those targeted by routine screening in primary care (Paniagua 2016). It means that IR usually starts as early as at the time when parameters of routine screening still may not show abnormal values. Effective detection of early IR could therefore contribute to identification of potential at-risk individuals. Similarly, effective and available objective assessment of IR in already identified at-risk individuals with MS could aid in predicting complication such as type 2 diabetes mellitus (T2DM).

A method for detecting IR that is easy to use in common clinical practice albeit still rather expensive for widespread use is homeostasis model assessment (HOMA). Its satisfactory correlation with the most accurate glucose clamp techniques has been confirmed by numerous studies. These simple mathematical models based on the knowledge of a single fasting glucose and insulin value reflect the level of resistance of peripheral tissues to insulin (HOMA-IR) or pancreatic beta cell function (HOMA- $\beta$ ) (Wallace et al. 2004).

Recently, there has been an increase in information in the literature about a relatively new predictive model called visceral adiposity index (VAI) that seems to be a reliable indicator of visceral adipose dysfunction; its increase is strongly associated with cardiometabolic risk (Amato et al. 2010). Similarly, atherogenic index of plasma (AIP) is associated with cardiometabolic risk. AIP reflects the true relationship between protective and atherogenic lipoprotein and is associated with the size of pre- and anti-atherogenic lipoprotein particles (Li et al. 2018). Unlike HOMA, both VAI and AIP may be calculated without knowing the patient's insulin concentration and only routine parameters are needed (serum lipids for AIP, plus basic anthropometric data for VAI).

The study aimed to assess the relationship between routinely used parameters and indirect IR markers, namely HOMA, with respect to the presence of MS, and to explore whether, and if so to what extent, VAI and AIP are associated with IR in those patients.

\section{Methods}

\section{Study subjects}

The study comprised two groups differing in the presence of MS. Those included in the MS group met the
International Diabetes Federation (IDF) criteria for diagnosing MS (Alberti et al. 2005), one of the widespread and commonly used definition of MS. Between March 2017 and September 2018, such individuals eligible for the study were selected in two general practitioners' offices in the Pardubice Region, Czech Republic. The MS group comprised 188 individuals (73 males and 115 females) with a mean age of 56 years. In these subjects, the below laboratory analyses were performed and, at the same time, their basic anthropometric parameters (height, weight, WC) and blood pressure (BP), as a mean of three resting recordings at a single visit, were measured. The obtained data were used to calculate Body Mass Index (BMI), HOMA-IR, HOMA- $\beta$, VAI and AIP for each participant. An equal number of controls without meeting the IDF criteria of MS was age- and sex-matched as par with the MS group. Those included in the control group were selected from the database of patients' first visits in an Outpatient center of the $3^{\text {rd }}$ Department of Internal Medicine, University Hospital Olomouc. None of the subjects of both groups was treated with oral antidiabetic drugs or insulin.

The study was conducted according to the principles stated in the Declaration of Helsinki. For being included in the study, all subjects signed informed consent forms after they were explained all information regarding the study.

\section{Laboratory analysis}

All laboratories participating in the study meet the same national accreditation. In all cases, the principles of proper laboratory practice were followed and the laboratories were under systematic intra- and inter- laboratory control of the accuracy of examinations.

In the MS group, venous blood was always sampled in the morning, after 12-hour fasting, in a single collection point of an accredited laboratory (MeDiLa Ltd., Pardubice). There, under standard conditions, the following biochemistry parameters were analyzed: glucose, total cholesterol (TC), low-density lipoprotein cholesterol (LDL-C), high-density lipoprotein cholesterol (HDL-C), triglycerides, uric acid (UA), alanine aminotransferase (ALT) and aspartate aminotransferase (AST). The above parameters were measured on the Architect c16000 analyzer (Abbott Laboratories, Illinois, USA) using fresh serum on the day of blood sampling. To determine serum insulin concentrations, serum was deep frozen within no more than 2 hours from blood 
sampling. The separated serum was stored at $-80{ }^{\circ} \mathrm{C}$ until assay. The insulin concentration analysis itself was carried out at the Department of Clinical Biochemistry and Diagnostics, University Hospital Hradec Králové on the Architect i1000SR analyzer (Abbott Laboratories) using chemiluminescent microparticle immunoassay.

In the control group, the same routine serum parameters were analyzed on the Cobas 8000 analyzer (Roche, Mannheim, Germany) on the day of blood sampling at the Department of Clinical Biochemistry, University Hospital Olomouc. Serum insulin concentrations were determined by the commercially available kits (Immunotech, Marseille, France) using specific antibodies by IRMA methods.

\section{Statistical analysis}

Statistical analyses were conducted with the $\mathrm{R}$ software environment ( $\mathrm{R}$ Foundation for Statistical
Computing, Austria; http://www.r-project.org/). All numerical variables were characterized with descriptive statistics. Correlations of selected variables were quantified with Pearson's correlation coefficient (r) and the level of significance (p) was determined. Multivariate linear regression was performed to examine the relationships between the parameters, including VAI and AIP, as regressors of the dependent variables HOMA-IR and HOMA- $\beta$. The results of regression analysis are presented in the form of linear coefficients $(\beta)$. P value of less than 0.05 indicates statistical significance. Extreme values of glucose and insulin were found and excluded using the inner and outer fences method. All variables of our interest followed normal or near-normal distribution. The following formulas were used to calculate the aforementioned indices (Wallace et al. 2004, Dobiasova and Frochlich 2001, Amato et al. 2010), units of the variables are given in Table 1.

$$
\begin{aligned}
& \text { HOMA-IR }=\frac{\text { glucose } \times \text { insulin }}{22.5} \\
& \text { HOMA- } \beta=\frac{\text { insulin } \times 20}{\text { glucose }-3.5} \\
& A I P=\log \frac{\text { triglycerides }}{H D L} \\
& V A I \text { in males }=\left(\frac{W C}{39.68+(1.88 \times B M I)}\right) \times\left(\frac{\text { triglycerides }}{1.03}\right) \times\left(\frac{1.31}{H D L}\right) \\
& V A I \text { in females }=\left(\frac{W C}{36.58+(1.89 \times B M I)}\right) \times\left(\frac{\text { triglycerides }}{0.81}\right) \times\left(\frac{1.52}{H D L}\right)
\end{aligned}
$$

\section{Results}

\section{Characteristics of the study population}

Basic metabolic and clinical characteristics are expressed as the mean of all values with the lower and upper limits of the $95 \%$ confidence interval (Table 1). It is apparent that most MS parameters were borderline or abnormal in the MS group. The mean values of WC (112.9 $\mathrm{cm}$ in males, $105.7 \mathrm{~cm}$ in females) were within the obesity range (according the IDF criteria) for both sexes. Those were individuals with visceral obesity only. As for other MS diagnostic criteria, glucose levels $\geq 5.6 \mathrm{mmol} / 1$ were commonly observed, namely in $56.2 \%$ of males and $49.6 \%$ of females. Glucose levels $\geq 7 \mathrm{mmol} / \mathrm{l}$ were present in $9.6 \%$ of males and $8.7 \%$ of females, respectively. As seen from Table 1, the mean systolic BP was slightly above the threshold for the applied MS criteria whereas the mean diastolic BP was below the threshold. Among the means of lipid parameters contained in the definition of MS, only triglycerides in males (1.81. mmol/l) were abnormal. It must be noted, however, that long-term antihypertensive and hypolipidemic therapy (both statins and fibrates) was widely used in the MS group. In the control group, means of all MS parameters were normal with the only exception of the borderline mean value of WC in females $(80.2 \mathrm{~cm})$. All tested parameters differed significantly between the groups except for TC and LDL-C. 
Table 1. Basic metabolic and clinical characteristics of subjects - mean value ( $95 \%$ confidence interval of the mean value)

\begin{tabular}{lccc}
\hline Characteristics & MS group & p-value & Controls \\
\hline N (males, females $)$ & $188(73,115)$ & - & $188(73,115)$ \\
Age $($ years $)$ & $56.26(54.24 ; 58.28)$ & 0.269 & $54.72(52.90 ; 56.55)$ \\
WC $(\mathrm{cm})$ & $108.47(106.27 ; 110.67)$ & $<0.001$ & $85.13(83.66 ; 86.59)$ \\
BMI $\left(\mathrm{kg} / \mathrm{m}^{2}\right)$ & $30.69(30.00 ; 31.38)$ & $<0.001$ & $25.31(24.83 ; 25.79)$ \\
Glucose $\left(\mathrm{mmol}^{2}\right)$ & $5.86(5.70 ; 6.03)$ & $<0.001$ & $4.99(4.90 ; 5.07)$ \\
Total cholesterol $(\mathrm{mmol} / \mathrm{l})$ & $5.41(5.25 ; 5.58)$ & 0.677 & $5.37(5.26 ; 5.49)$ \\
HDL-C $(\mathrm{mmol} / \mathrm{l})$ & $1.29(1.25 ; 1.34)$ & $<0.001$ & $1.56(1.51 ; 1.62)$ \\
LDL-C $(\mathrm{mmol} / \mathrm{l})$ & $3.36(3.21 ; 3.50)$ & 0.168 & $3.23(3.11 ; 3.34)$ \\
Triglycerides $(\mathrm{mmol} / \mathrm{l})$ & $1.51(1.40 ; 1.63)$ & 0.007 & $1.32(1.24 ; 1.41)$ \\
Uric acid $(\mathrm{mmol} / \mathrm{l})$ & $340.87(329.87 ; 351.87)$ & $<0.001$ & $281.56(272.89 ; 290.24)$ \\
ALT $(\mu \mathrm{kat} / \mathrm{l})$ & $0.57(0.54 ; 0.60)$ & $<0.001$ & $0.42(0.40 ; 0.45)$ \\
AST $(\mu \mathrm{kat} / \mathrm{l})$ & $0.42(0.40 ; 0.44)$ & $<0.001$ & $0.35(0.33 ; 0.37)$ \\
Insulin $(\mathrm{mIU} / \mathrm{l})$ & $12.86(11.27 ; 14.46)$ & $<0.001$ & $7.17(6.62 ; 7.73)$ \\
BP systolic $(\mathrm{mmHg})$ & $141.35(139.09 ; 143.60)$ & $<0.001$ & $126.80(125.98 ; 127.62)$ \\
BP diastolic $(\mathrm{mmHg})$ & $82.00(80.59 ; 83.41)$ & $<0.001$ & $76.54(75.65 ; 77.42)$ \\
HOMA-IR & $2.72(2.48 ; 2.98)$ & $<0.001$ & $1.37(1.26 ; 1.49)$ \\
HOMA- $\beta$ & $97.48(89.38 ; 106.32)$ & $<0.001$ & $89.82(82.26 ; 98.08)$ \\
VAI & $2.19(2.00 ; 2.40)$ & $<0.001$ & $1.40(1.28 ; 1.52)$ \\
AIP & $0.08(0.04 ; 0.12)$ & $<0.001$ & $-0.06(-0.10 ;-0.02)$ \\
\hline
\end{tabular}

MS - metabolic syndrome; WC - waist circumference; BMI - body mass index; HDL-C - high-density lipoprotein cholesterol; LDL-C low-density lipoprotein cholesterol; ALT - alanine aminotransferase; AST - aspartate aminotransferase; BP - blood pressure; HOMA homeostasis model assessment; IR - insulin resistance; VAI - visceral adiposity index; AIP - atherogenic index of plasma

Table 2. Pearson's correlation coefficient ( $r$ ) between several investigated indices and other tested parameters in the MS group/control group

\begin{tabular}{|c|c|c|c|c|c|c|}
\hline Characteristics & Glucose & Insulin & HOMA-IR & НОМА- $\beta$ & VAI & AIP \\
\hline$W C$ & $0.19 * / 0.22 *$ & $0.56^{* *} / 0.35^{* *}$ & $0.57 * * / 0.36 * *$ & $0.39 * * / 0.06$ & $0.22 * / 0.26 * *$ & $0.30 * * / 0.4 * *$ \\
\hline$B M I$ & $0.10 / 0.18^{*}$ & $0.43 * * / 0.37 * *$ & $0.42 * * / 0.37 * *$ & $0.33 * * / 0.07$ & $0.16^{*} / 0.16^{*}$ & $0.23 * / 0.29 * *$ \\
\hline Total cholesterol & $-0.05 / 0.01^{*}$ & $0.08 / 0.24 * *$ & $0.08 / 0.26^{* *}$ & $0.06 / 0.05$ & $0.21 * / 0.21^{*}$ & $0.16 * / 0.19 *$ \\
\hline$H D L-C$ & $-0.02 /-0.05$ & $-0.17 * /-0.17 *$ & $-0.15^{*} /-0.15^{*}$ & $-0.22 * / 0.02$ & $-0.42 * * /-0.47 * *$ & $-0.64 * * /-0.69 * *$ \\
\hline$L D L-C$ & $-0.02 / 0.16^{*}$ & $0.17 * / 0.18 *$ & $0.17 * / 0.20 *$ & $0.13 / 0.01$ & $0.28 * * / 0.17 *$ & $0.29 * * / 0.20 *$ \\
\hline Triglycerides & $0.06 /-0.01$ & $0.19 * / 0.25 * *$ & $0.19 * / 0.21 *$ & $0.14 / 0.13$ & $0.92 * * / 0.94 * *$ & $0.79 * * / 0.82 * *$ \\
\hline Uric acid & $0.01 / 0.14$ & $0.20 * / 0.46 * *$ & $0.18 * / 0.44 * *$ & $0.17 * / 0.19 *$ & $0.30 * * / 0.16^{*}$ & $0.41 * * / 0.31 * *$ \\
\hline$A L T$ & $0.03 / 0.22 *$ & $0.44 * / 0.56 * *$ & $0.41 * * / 0.57 * *$ & $0.36 * * / 0.19 *$ & $0.22 * / 0.17 *$ & $0.30 * * / 0.27 * *$ \\
\hline$A S T$ & $0.10 / 0.21 *$ & $0.31 / 0.55^{* *}$ & $0.31 * * / 0.56 * *$ & $0.21 * / 0.18 *$ & $0.17 * / 0.16^{*}$ & $0.24 * / 0.27 * *$ \\
\hline BP systolic & $0.33 * * / 0.05$ & $0.32 * * / 0.18 *$ & $0.37 * * / 0.17 *$ & $0.12 / 0.10$ & $0.32 * * / 0.06$ & $0.32 * * / 0.10$ \\
\hline BP diastolic & $0.22 * / 0.02$ & $0.31 * * / 0.22 *$ & $0.34 * * / 0.2 *$ & $0.16^{*} / 0.18^{*}$ & $0.29 * * / 0.03$ & $0.33 * * / 0.09$ \\
\hline$V A I$ & $0.08 /-0.01$ & $0.24 * * / 0.26^{* *}$ & $0.24 * * / 0.21 *$ & $0.20 * / 0.12$ & - & $0.87 * * / 0.86^{* *}$ \\
\hline$A I P$ & $0.09 / 0.05$ & $0.27 * * / 0.26^{* *}$ & $0.27 * * / 0.26 *$ & $0.25 * * / 0.06$ & $0.87 * * / 0.86^{* *}$ & - \\
\hline
\end{tabular}

* $\mathrm{p}<0.05, * * \mathrm{p}<0.001, \mathrm{MS}$ - metabolic syndrome; HOMA - homeostasis model assessment; IR - insulin resistance; VAI - visceral adiposity index; AIP - atherogenic index of plasma; WC - waist circumference; BMI - body mass index; HDL-C - high-density lipoprotein cholesterol; LDL-C - low-density lipoprotein cholesterol; ALT - alanine aminotransferase; AST - aspartate aminotransferase; BP - blood pressure 

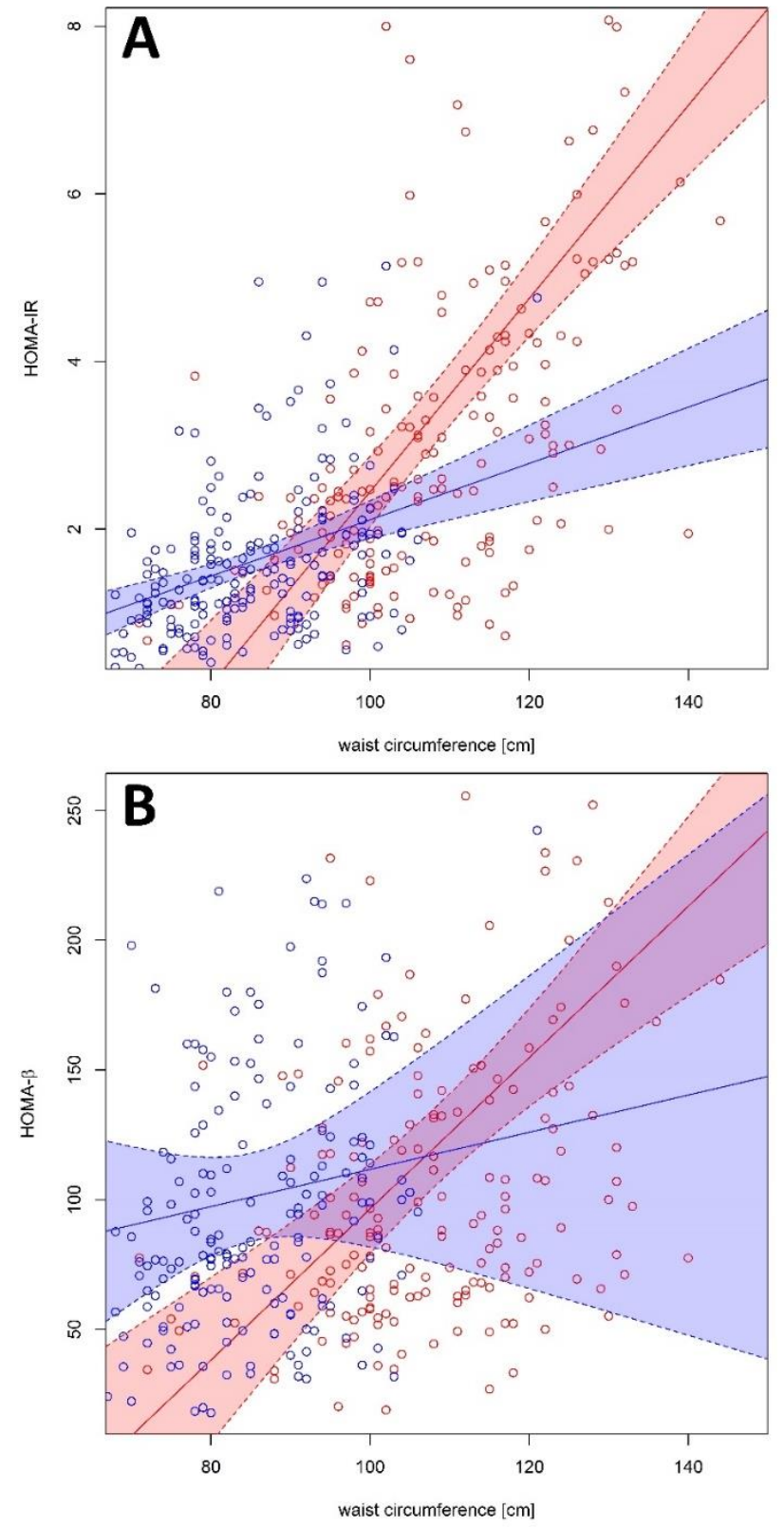

Fig. 1. Scatter diagrams showing correlations of waist circumference with HOMA-IR (A) and HOMA- $\beta$ (B) in both the MS group (red) and the control group (blue) with $95 \%$ confidence bands for the medians of variables on the $y$-axis for a given variable on the $x$-axis. The comparison of both study groups shows that correlations are stronger in subjects with metabolic syndrome, especially in case of HOMA-IR (A). The association of waist circumference with HOMA- $\beta$ in the controls (B) is among others clearly the least significant. HOMA - homeostasis model assessment; IR - insulin resistance

\section{Correlation analysis}

In the MS group, the highest correlation coefficients were noted between $\mathrm{WC}$ and IR markers, namely correlations of WC with HOMA-IR ( $\mathrm{r}=0.57)$, HOMA- $\beta \quad(r=0.39)$ and insulin concentration alone $(\mathrm{r}=0.56)$, as seen from Table 2 and Fig. 1. However, WC was only weakly correlated with glucose alone $(\mathrm{r}=0.19)$.
Similar correlations were found for BMI, with correlation coefficients being generally lower than in case of WC. Both HOMA and insulin were also moderately correlated with liver transaminases, the strongest being correlations of ALT with insulin concentration $(r=0.44)$ and HOMA-IR $(r=0.41)$. Statistically significant were also correlations of systolic and diastolic BP with HOMA-IR ( $\mathrm{r}=0.37$ and $\mathrm{r}=0.34$, respectively), insulin concentration alone ( $r=0.32$ and $r=0.31$, respectively) and glucose $(r=0.33$ and $r=0.22$, respectively). As for the parameters not directly associated with IR, there were statistically highly significant $(\mathrm{p}<0.001)$ correlations of WC and BMI with both systolic $(r=0.42$ and $r=0.31$, respectively) and diastolic $\mathrm{BP}(\mathrm{r}=0.45$ and $\mathrm{r}=0.36$, respectively) and HDL-C ( $\mathrm{r}=-0.34$ and $\mathrm{r}=-0.32$, respectively). As expected, correlations of VAI and AIP with parameters included in their calculations were strong and statistically significant. As for the parameters not included in the calculations of VAI and AIP, the strongest correlations were found in BP and UA, specifically the strongest between AIP and UA ( $r=0.41)$.

In the control group, an association of the anthropometric indices with HOMA was generally weaker than in the MS group, without an apparent difference between WC and BMI (Table 2). There were moderate correlations of both insulin and HOMA-IR with ALT and AST, respectively, with the highest correlation coefficient noted between HOMA-IR and ALT ( $r=0.57$ ) (Fig. 2). Also notable was an association of UA with both insulin $(\mathrm{r}=0.46)$ and HOMA-IR ( $\mathrm{r}=0.44)$. Correlation coefficients of HOMA$\beta$ were generally very low (Table 2 ).

\section{Regression analysis}

The impact of individual HOMA predictors is expressed with multivariate linear regression models that use linear coefficients $(\beta)$ to show the effects of 1-point increases of these predictors on HOMA (Table 3). As for HOMA-IR, there were statistically significant results for its relationships with $\mathrm{WC}$, ALT and systolic BP. For HOMA- $\beta$, the statistically significant predictors were WC and ALT; unlike HOMA-IR, the result for an inverse association between HOMA- $\beta$ and age was statistically significant. The presence of MS was a significant predictor of both HOMA-IR and HOMA- $\beta$. For example, if WC increases by 1 point, HOMA-IR and HOMA- $\beta$ will increase by a mean of $0.1(\mathrm{p}<0.001)$ and $1.97(\mathrm{p}=0.032)$, respectively. If ALT increases by 1 point, HOMA-IR and HOMA- $\beta$ will increase by $2.28(\mathrm{p}<0.001)$ and 99.49 $(p=0.004)$, respectively. With each year of age HOMA- $\beta$ will decrease by a mean of $1.31(\mathrm{p}=0.004)$. 


\section{Discussion}

The obtained results of both correlation and regression analyses demonstrate strong relationships between WC and IR markers in individuals with MS. However, the same regression analysis results were not observed for BMI. The ability of WC to predict IR may be beneficial, mainly from a primary health care practitioner's perspective, given the ease with which WC is measured. Our results are similar to those of a cross-
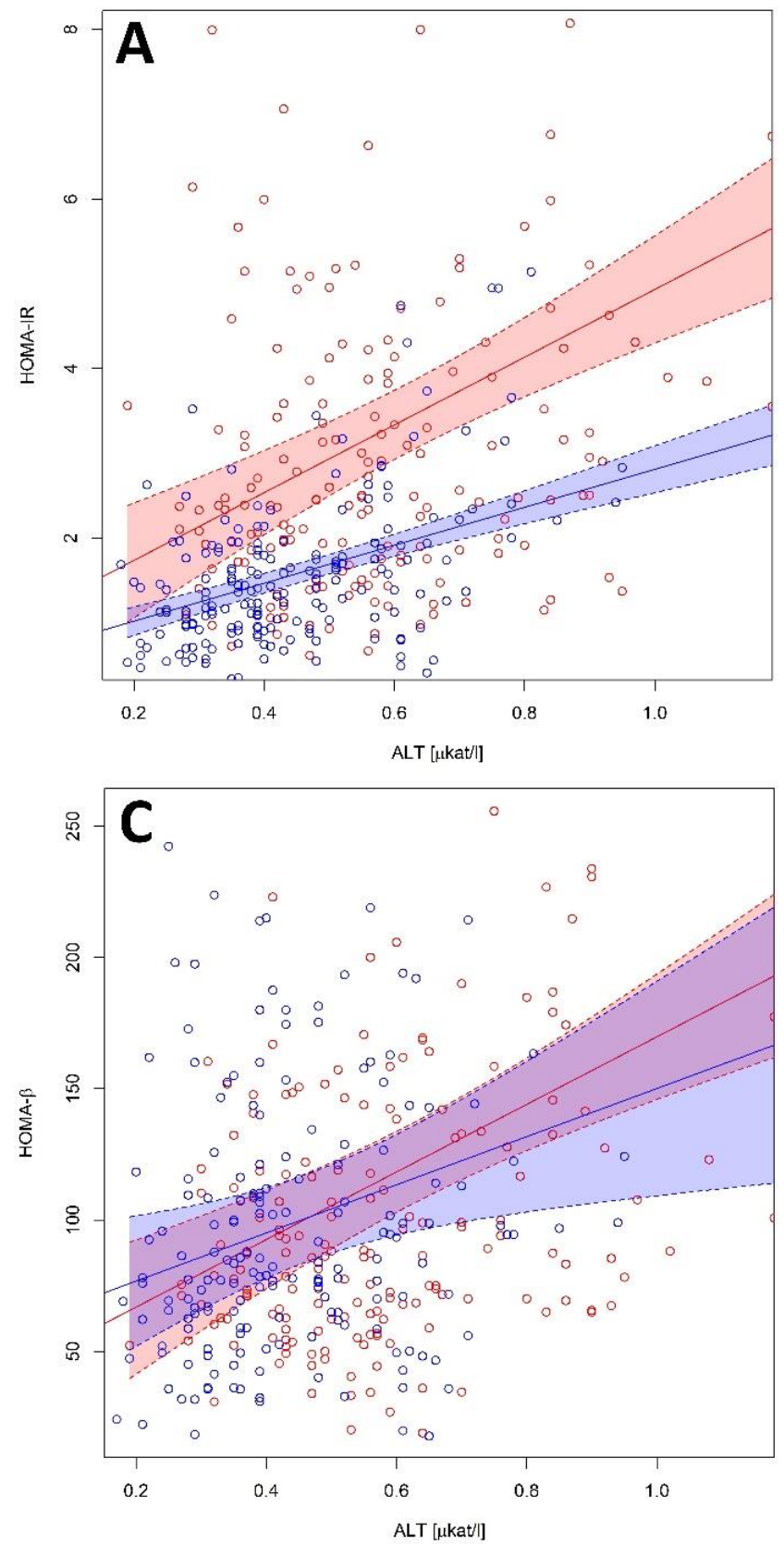

sectional study by Huang et al. (2012) who used regression analysis of 144 individuals with T2DM to show that unlike BMI, WC is a statistically significant predictor of HOMA-IR. Although a cross-sectional study of 140 healthy individuals by Kurniawan et al. (2018) found, consistently with the present study, a stronger correlation of HOMA-IR with WC $(r=0.46$, $p<0.01)$ than with BMI $(\mathrm{r}=0.39, \mathrm{p}<0.01)$, regression analysis proved BMI to be a stronger predictor of HOMA-IR than WC.
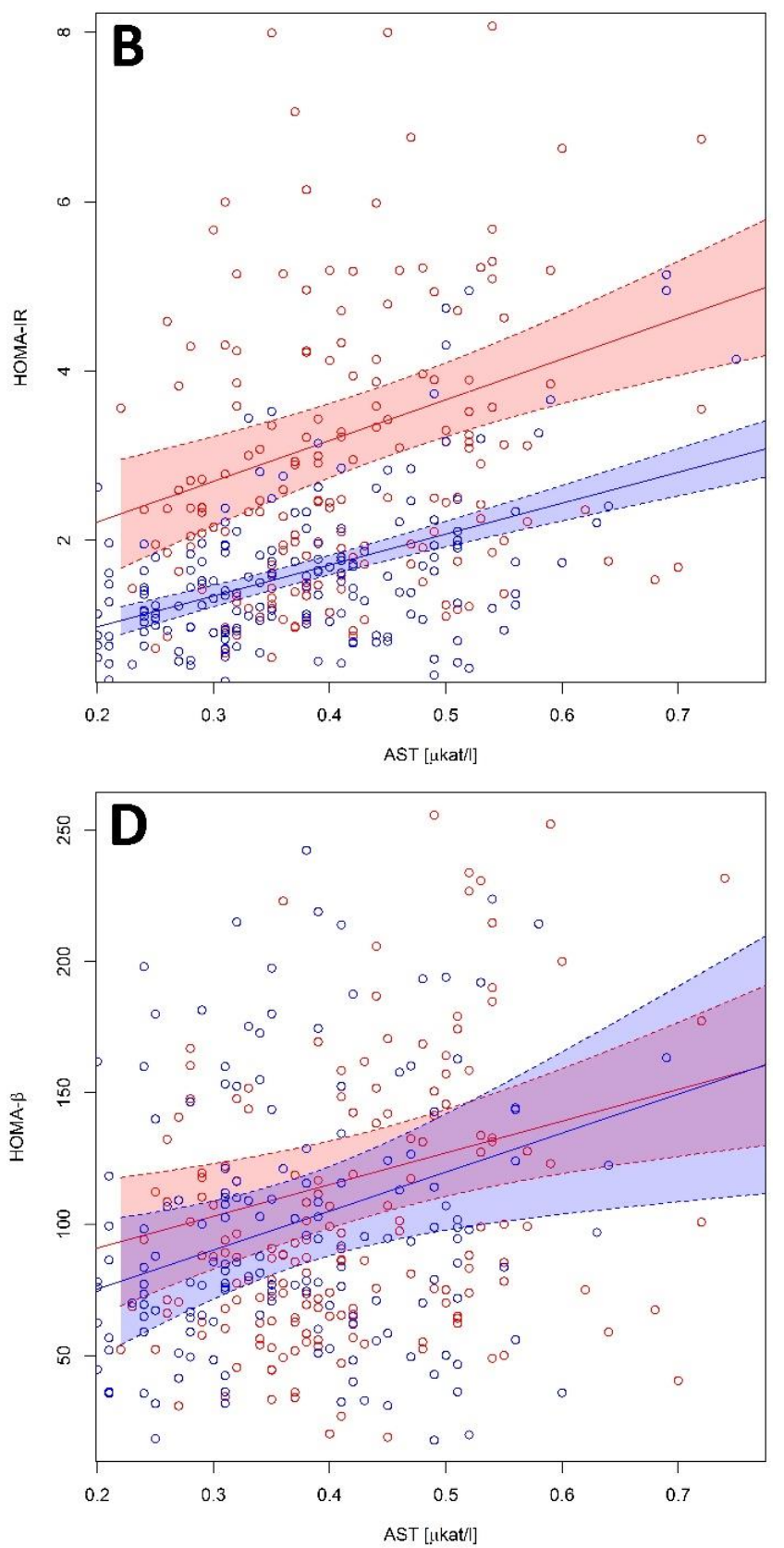

Fig. 2. Scatter diagrams showing correlations of ALT with HOMA-IR (A) and HOMA- $\beta$ (C) as well as AST with HOMA-IR (B) and HOMA- $\beta$ (D) in both the MS group (red) and the control group (blue) with $95 \%$ confidence bands for the medians of variables on the $y$-axis for a given variable on the $x$-axis. Displayed associations of a particular HOMA are similar for both transaminases in the same study group, however, there are apparent differences between the groups. Correlations are generally stronger and more significant in case of HOMA-IR (A, B). HOMA - homeostasis model assessment; IR - insulin resistance; ALT - alanine aminotransferase; AST aspartate aminotransferase 
Table 3. Effects of every 1-point increase of independent variables on the dependent variables HOMA-IR and HOMA- $\beta$

\begin{tabular}{|c|c|c|c|c|c|c|c|c|}
\hline \multirow{2}{*}{$\begin{array}{l}\text { Independent } \\
\text { variables }\end{array}$} & \multicolumn{2}{|c|}{ Linear coefficient $(\beta)$} & \multicolumn{2}{|c|}{ Standard error } & \multicolumn{2}{|c|}{ t-value } & \multicolumn{2}{|c|}{ p-value } \\
\hline & HOMA-IR & НОМА- $\beta$ & HOMA-IR & НОМА- $\beta$ & HOMA-IR & НОМА- $\beta$ & HOMA-IR & НОМА- $\beta$ \\
\hline Intercept & -10.44 & -147.1 & 1.34 & 81.11 & -7.81 & -1.81 & $<0.001$ & 0.071 \\
\hline$M S+$ & 0.73 & -43.92 & 0.28 & 17.26 & 2.58 & -2.55 & 0.010 & 0.011 \\
\hline Age & -0.02 & -1.31 & 0.01 & 0.45 & -1.47 & -2.9 & 0.139 & 0.004 \\
\hline$W C$ & 0.10 & 1.97 & 0.02 & 0.91 & 6.34 & 2.16 & $<0.001$ & 0.032 \\
\hline$B M I$ & -0.06 & -0.04 & 0.04 & 2.47 & -1.45 & -0.02 & 0.147 & 0.990 \\
\hline Total cholesterol & -0.21 & 5.88 & 0.25 & 15.05 & -0.84 & 0.39 & 0.402 & 0.696 \\
\hline$H D L-C$ & -1.05 & 31.78 & 0.58 & 35.28 & -1.82 & 0.90 & 0.070 & 0.368 \\
\hline$L D L-C$ & 0.38 & -4.73 & 0.26 & 15.51 & 1.50 & -0.31 & 0.135 & 0.761 \\
\hline Triglycerides & -0.15 & -14.17 & 0.33 & 19.81 & -0.45 & -0.72 & 0.651 & 0.475 \\
\hline Uric acid & -0.01 & 0.10 & 0.01 & 0.10 & -1.18 & 0.99 & 0.239 & 0.321 \\
\hline$A L T$ & 2.28 & 99.49 & 0.57 & 34.54 & 4.02 & 2.88 & $<0.001$ & 0.004 \\
\hline$A S T$ & 0.12 & -46.93 & 0.91 & 55.42 & 0.14 & -0.85 & 0.888 & 0.398 \\
\hline BP systolic & 0.04 & -0.31 & 0.01 & 0.64 & 3.69 & -0.49 & $<0.001$ & 0.628 \\
\hline BP diastolic & -0.01 & 0.72 & 0.02 & 0.96 & -0.87 & 0.75 & 0.385 & 0.452 \\
\hline$V A I$ & 0.10 & 11.98 & 0.23 & 13.88 & 0.45 & 0.86 & 0.656 & 0.389 \\
\hline$A I P$ & 0.79 & 27.74 & 0.96 & 58.18 & 0.83 & 0.48 & 0.410 & 0.634 \\
\hline
\end{tabular}

HOMA - homeostasis model assessment; IR - insulin resistance; MS+ - meeting the International Diabetes Federation criteria (2005) for diagnosing metabolic syndrome; WC - waist circumference; BMI - body mass index; HDL-C - high-density lipoprotein cholesterol; LDL-C - low-density lipoprotein cholesterol; ALT - alanine aminotransferase; AST - aspartate aminotransferase; BP - blood pressure; VAI visceral adiposity index; AIP - atherogenic index of plasma

As demonstrated by our results, stronger and more statistically significant correlations between WC and insulin or HOMA are in subjects with MS than in metabolically healthy subjects (Table 2, Fig. 1).

Uniform reference values for HOMA have not been defined as yet. Tang et al. (2015) state that HOMA cut-offs vary, depending on geographical area and the studied population. For various European regions, HOMA-IR identifying established IR has been reported to range from 2.0 to 3.8 . In the present study, the mean HOMA-IR was 2.72 in the MS group, differing significantly from the mean HOMA-IR of 1.37 in the control group. In a sample of 3636 males, Ying et al. (2011) demonstrated that HOMA-IR value increases as the number of MS components increases. For males with 3 or more MS components, HOMA-IR was 2.64. In our study, the mean HOMA-IR in males with MS was 2.98 . Recent decades have witnessed an increase in the prevalence of child MS. HOMA-IR cut-offs in children differ from those in adults (Pastucha et al. 2013).

In their cross-sectional study of 50 MS patients (IDF-defined as in the present study) and 24 healthy controls, Garg et al. (2011) found lower HOMA- $\beta$ in MS patients than in controls (66.80 vs. 144.27). In our study, the situation was opposite with the mean HOMA- $\beta$ of 97.48 in the MS group and 89.82 in controls, respectively. In other words, insulin secretion of MS subjects was less altered in the present study than in the analysis by Garg et al. (2011), however, our controls exhibited much lower insulin secretion in comparison with the analysis. A Mexican study of 190 individuals meeting the MS criteria (NCEP ATP III) showed a mean HOMA- $\beta$ of 115.2 and progressive deterioration of $\beta$-cell function (HOMA- $\beta$ decrease) as the number of components of MS increased. In individuals with MS, the mean insulin concentration was $11.9 \mathrm{mIU} / \mathrm{l}$ (Baez-Duarte et al. 2010). In the present study, significant positive predictors of HOMA- $\beta$ were WC and ALT. Therefore, the participants probably had a persistent functional reserve capacity of the endocrine part of pancreas, with excess insulin-dependent visceral adipose tissue inducing increased insulin production. The inverse association of HOMA- $\beta$ with age confirms an unsurprising decrease in pancreatic endocrine function throughout the life span (Hirose et al. 2016).

The liver plays a central role in the systemic 
regulation of glucose and lipid metabolism and aberrant hepatic insulin action is thought to be a primary driver of IR. In pathologic states, insulin fails to appropriately regulate hepatic metabolism, leading to excess production of glucose despite accelerated rates of lipid synthesis. As a consequence, IR disorders such as obesity and T2DM are closely linked to nonalcoholic fatty liver disease (NAFLD) (Santoleri and Titchenell 2019, Poledne et al. 2015). Markers of liver function, specifically ALT, predict incident T2DM in various populations. In a study of 1309 healthy individuals with IR quantified by clamp techniques, increased ALT was a biomarker of IR with concomitant increased insulin secretion and decreased hepatic insulin clearance (Bonnet et al. 2011). This is consistent with the positive associations of ALT with HOMA-IR as well as of ALT with HOMA- $\beta$ in the present study. A study of 1732 adults aged 18-23 years with normal weight by Simental-Mendía et al. (2017) concluded that IR was significantly associated with elevated ALT levels but not with elevated AST levels using logistic regression analysis adjusted by age, sex, waist circumference and BMI. This corresponds with results of the regression model in the present study.

It is known that AIP is associated with obesity and increased cardiovascular risk. Pearson's correlation analysis of 5351 middle-aged males from Southeastern China revealed that AIP was positively correlated with WC ( $\mathrm{r}=0.37, \mathrm{p}<0.001)$ (Shen et al. 2018). In their study, Li et al. (2018) divided 2523 individuals with T2DM who had not been treated with lipid-lowering drugs into tertiles based on their AIP values. There was a significant increase in HOMA-IR between the tertiles. Multiple logistic regression analysis showed that WC, HOMA-IR, fasting glucose, systolic BP and UA were independent risk factors for AIP. The present study documented several rather weak statistically highly significant correlations of AIP (Table 2), however, regression analysis did not confirm the ability of AIP to predict HOMA.

A cross-sectional study including 1834 Chinese adults with normal WC assessed the correlation between VAI and HOMA-IR. VAI gradually increased across
HOMA-IR quartiles and correlation analysis showed that VAI was positively related to HOMA-IR. Logistic regression analysis indicated that VAI elevation was the main risk factor for the increased HOMA-IR in both genders (Ji et al. 2017). In the present study, regression analysis failed to confirm a significant association between HOMA-IR and VAI and only showed statistically significant correlations of VAI with LDL-C, UA and BP in the MS group (Table 2).

The study has certain limitations. One is the fact that it used laboratory data obtained by single measurement, causing possible bias due to natural intraindividual variability of the analyzed parameters, as is well known, for instance, in fasting glucose (Pasqualetti et al. 2017). Another limitation is that common long-term antihypertensive and hypolipidemic therapy was widespread in the study population (especially in the MS group), affecting plasma lipid levels and BP values.

In conclusion, correlation and regression analysis confirmed significant associations between HOMA and some routinely examined parameters. In addition to systolic BP, significant predictors for HOMA-IR were also ALT and WC, clearly the most significant predictor. The association of WC with HOMA-IR increases with a number of metabolic disorders present as indicated by the difference of correlation coefficients between the studied groups. Thus, targeted assessment of these routine and easily available parameters may be used to identify IR without the need to detect insulin. Primarily, the predictive ability of WC must not be underestimated. The important predictors of HOMA- $\beta$ were WC, ALT and age. After further verification, these findings may considerably contribute to preventive measures, particularly in primary care.

\section{Conflict of Interest}

There is no conflict of interest.

\section{Acknowledgements}

This work was supported by IGA LF 2017016 and MZ ČR - RVO FNOL 00098892.

\section{References}

ALBERTI KG, ZIMMET P, SHAW J: The metabolic syndrome - a new worldwide definition. Lancet 366: 1059-1062, 2005.

AMATO MC, GIORDANO C, GALIA M, CRISCIMANNA A, VITABILE S, MIDIRI M, GALLUZZO A: Visceral Adiposity Index A reliable indicator of visceral fat function associated with cardiometabolic risk. Diabetes Care 33: 920-922, 2010. 
BAEZ-DUARTE BG, SÁNCHEZ-GUILLÉN MDC, PÉREZ-FUENTES R, ZAMORA-GINEZ I, LEON-CHAVEZ BA, REVILLA-MONSALVE C, ISLAS-ANDRADE S: $\beta$-cell function is associated with metabolic syndrome in Mexican subjects. Diabetes Metab Syndr Obes 3: 301-309, 2010.

BONNET F, DUCLUZEAU PH, GASTALDELLI A, LAVILLE M, ANDERWALD CH, KONRAD T, MARI A, BALKAU B: Liver enzymes are associated with hepatic insulin resistance, insulin secretion, and glucagon concentration in healthy men and women. Diabetes 60: 1660-1667, 2011.

DOBIASOVA M, FROHLICH J: The plasma parameter log (TG/HDL-C) as an atherogenic index: correlation with lipoprotein particle size and esterification rate inapob-lipoprotein-depleted plasma (FERHDL). Clin Bioch 34: 583-588, 2001.

GARG MK, DUTTA MK, MAHALLE N: Study of beta-cell function (by HOMA model) in metabolic syndrome. Indian J Endocrinol Metab 15: S44-49, 2011.

HIROSE H, TAKAYAMA M, IWAO Y, KAWABE H: Effects of aging on visceral and subcutaneous fat areas and on homeostasis model assessment of insulin resistance and insulin secretion capacity in a comprehensive health checkup. J Atheroscler Thromb 23: 207-215, 2016.

HUANG LH, LIAO YL, HSU CH: Waist circumference is a better predictor than body mass index of insulin resistance in type 2 diabetes. Obes Res Clin Pract 6: e263-346, 2012.

JI B, QU H, WANG H, WEI H, DENG H: Association between the visceral adiposity index and homeostatic model assessment of insulin resistance in participants with normal waist circumference. Angiology 68: 716-721, 2017.

KASSI E, PERVANIDOU P, KALTSAS G, CHROUSOS G: Metabolic syndrome: definitions and controversies. BMC Med 9: 48, 2011.

KURNIAWAN LB, BAHRUN U, HATTA M, ARIF M: Body mass, total body fat percentage, and visceral fat level predict insulin resistance better than waist circumference and body mass index in healthy young male adults in Indonesia. J Clin Med 7: E96, 2018.

LI Z, HUANG Q, SUN L, BAO T, DAI Z: Atherogenic index in type 2 diabetes and its relationship with chronic microvascular complications. Int J Endocrinol 2018: 1765835, 2018.

MATLOCH Z, KOTULÁK T, HALUZÍK M: The role of epicardial adipose tissue in heart disease. Physiol Res $\mathbf{6 5}$ : 23-32, 2016.

PANIAGUA JA: Nutrition, insulin resistance and dysfunctional adipose tissue determine the different components of metabolic syndrome. World J Diabetes 7: 483-514, 2016.

PASQUALETTI S, BRAGA F, PANTEGHINI M: Pre-analytical and analytical aspects affecting clinical reliability of plasma glucose results. Clin Biochem 50: 587-594, 2017.

PASTUCHA D, FILIPCIKOVA R, HORAKOVA D, RADOVA L, MARINOV Z, MALINCIKOVA J, KOCVRLICH M, HORAK S, BEZDICKOVA M, DOBIAS M: The incidence of metabolic syndrome in obese Czech children: the importance of early detection of insulin resistance using homeostatic indexes HOMA-IR and QUICKI. Physiol Res 62: 277-283, 2013.

POLEDNE R, KRALOVA LESNA I, CEJKOVA S: Adipose tissue and atherosclerosis. Physiol Res 64 (Suppl. 3): S395-S402, 2015.

SANTOLERI D, TITCHENELL PM: Resolving the Paradox of Hepatic Insulin Resistance. Cell Mol Gastroenterol Hepatol 7: 447-456, 2019.

SHEN SW, LU Y, LI F, YANG CJ, FENG YB, LI HW, YAO WF, SHEN ZH: Atherogenic index of plasma is an effective index for estimating abdominal obesity. Lipids Health Dis 17: 11, 2018.

SIMENTAL-MENDÍA LE, RODRÍGUEZ-MORÁN M, GÓMEZ-DÍAZ R, WACHER NH, RODRÍGUEZHERNÁNDEZ H, GUERRERO-ROMERO F: Insulin resistance is associated with elevated transaminases and low aspartate aminotransferase/alanine aminotransferase ratio in young adults with normal weight. Eur $J$ Gastroenterol Hepatol 29: 435-440, 2017.

TANG Q, LI X, SONG P, XU L: Optimal cut-off values for the homeostasis model assessment of insulin resistance (HOMA-IR) and pre-diabetes screening: Developments in research and prospects for the future. Drug Discov Ther 9: 380-385, 2015.

WALLACE TM, LEVY JC, MATTHEWS DR: Use and abuse of HOMA modeling. Diabetes Care 27: 1487-1495, 2004. 
YING X, SONG Z, ZHAO C, JIANG Y: Association between homeostasis model assessment of insulin resistance (HOMA-IR) and components of metabolic syndrome in young Chinese men. Iran J Public Health 40: 1-5, 2011. 